Voix et Images

voixetimages

\title{
Jean-Claude Germain : Au théâtre d'aujourd'hui. Entretien
}

\section{Bernard Andrès et Yves Lacroix}

Volume 6, numéro 2, hiver 1981

Jean-Claude Germain

URI : https://id.erudit.org/iderudit/200261ar

DOI : https://doi.org/10.7202/200261ar

Aller au sommaire du numéro

\section{Éditeur(s)}

Les Presses de l'Université du Québec

\section{ISSN}

0318-9201 (imprimé)

1705-933X (numérique)

Découvrir la revue

\section{Citer ce document}

Andrès, B. \& Lacroix, Y. (1981). Jean-Claude Germain : Au théâtre d'aujourd'hui. Entretien. Voix et Images, 6(2), 169-187. https://doi.org/10.7202/200261ar d'utilisation que vous pouvez consulter en ligne.

https://apropos.erudit.org/fr/usagers/politique-dutilisation/ 


\title{
Jean-Claude Germain: Au théâtre d'aujourd'hui
}

\author{
Entrellen/Bernard Andres et Yves Lacroix
}

\section{Introduction}

Yves Lacroix et moi-mêrne l'avons rencontré le 23 octobre 1980 dans son bureau niché au dernier étage du fameux petit théatre de la rue Papineau. Ce n'est qu'apress avoir traversé une enfilade de couloirs, d'escaliers et de pièces encombrées de décors passés et à venir, dans l'odeur de plâtre et de colle émanant de l'atelier voisin, que nous avons entrepris cette discussion avec l'auteur qui fêtait l'an dernier le dixième anniversaire du Théâtre d'Aujourd'hui. Sur son bureau, une statuette de Louis Cyr lancant un regard complice au bateleur malicieux du théâtre québécois: Jean-Claude Germain.

Bernard Andres.

\section{Theorie et pratique}

B.A. Deux images circulent ordinairement dans le milieu. Chez les littéraires, c'est le Germain praticien, homme à tout faire du Thétre d'Aujourd'hui : directeur-auteur-metteur en scène. Celte image aussi connue des gens de théâtre, est relayée chez eux (surtout chez les jeunes comédiens que tu as formés), par une autre perception de l'homme: le Germain professeur (tu enseignes à l'École Nationale de Théâtre) le théoricien dans un certain sens, voire l'universitaire (c'est le point de vue de Louisette Dussault '1. Si la seconde image est moins connue, c'est que toi-même, tu as tendance à favoriser celle du praticien, ton discours étant parfois teinté d'anti-intellectualisme quand tu parles de thérie. S'agit-il de fausses images? En favorises-tu réellement une des deux?

J.-C.G. Je crois que d'abord, pour moi, il n'y a pas de rupture entre le praticien et le théoricien parce qu'au niveau thétral, la théorie nait de la pratique. C'est-à-dire qu'à partir de ce qu'on essaie, a priori, au niveau de la pratique, inévitablement, une théorie s'élabore, a postériori. Tous les directeurs de théâtre, les metteurs en scène, les auteurs qui ont une certaine protique thétrale deviennent éventuellement des théoriciens de leur propre ceuvre; à la limite Barillet et Grédy qui sont 
des auteurs de boulevard, sont des théoriciens du boulevard à partir de leur pratıque du succès facile. La théorie n'est peut-tre pas plus intéressante que le résultat, mais c'est une théorie quand mème. Donc, pour moi, il n'y a pas de confusion entre les deux: la théorie et la pratique sont l'envers et l'endroit d'un même regard critique. Quant à mes relations avec I'Université, elles sont liées au fait que j'y suis allé à une époque donnée, précise, vers la fin des années cinquante, où l'université se définissait comme l'ailleurs. A l'époque, j'étais en histoire et toutes les thèses étaient faites sur le Moyen Áge, nous étions appelés à devenir des experts du Moyen Age et ce n'est que deux ou trois ans après que j'ai quitté l'université que le directeur du département. Michel Brunet, est parvenu à imposer que les thèses de maitrise soient obligatoirement faites sur des sujets québécois. ce qui était l'évidence, puisqu'en histoire, il est impossible de concevoir des ouvrages de synthèse sans qu'il existe, au préalable, tout un ensemble d'études spécifiques et de monographies sur des sujets d'intérêt limité. Donc, autant le théâtre québécois a évolué, autant. je crois, l'université a évolué ; autant elle était à une époque donnée et, particulièrement dans les études littéraires, complètement axée sur un ailleurs, un impossible ailleurs: c'était «anywhere but... Québec ", il y a eu transformation, il y a eu un retour, ou plutôt un départ, vers l'étude du matériau québécois; et les universitaires ont eu la méme réaction que nous au théâtre, ils ont découvert que c' était extraordinaire de se retrouver en terrain vierge et de se rendre compte qu'd chaque fois qu'ils ouvraient une boîte de documents inédits, il $y$ en avait toujours une autre en-dessous. Actuellement, il y a une floraison d'études passionnantes, autant littéraires qu'historiques, et c'est ce qui me permet d'avoir une relation différente avec le monde universitaire. L'image que j'ai combattu de l'université est celle que j'en avais gardé; avec la nouvelle, il est au moins possible de dialoguer. Par exemple, au niyeau de l'histoire du thétre, l'an dernier, j'ai participé à un colloque organisé par la Société d’Histoire du théâtre du théâtre au Québec qui regroupe des universitaires, c'est une réalité nouvelle extrêmement stimulante quand on pense qu'avant, c'était presque un sacrilege de reconnaître qu'il y avait une histoire du théâtre au Québec.

B.A. De par ta formation d'historien, tu $t^{\prime}$ intéresses toi-mème à ce domaine. Tu participes à des colloques dans le cadre des Sociétés Savantes. Tu as fait une série d'émissions de radio sur l'histoire du théâtre au Québec. Est-ce que ces émissions se poursuivent ?

J.-C.G. Non, ça a marché pendant un an et il semble que Radio-Canada s'en soit lassé... avant les auditeurs I

B.A. C'est une recherche que tu fais toi-méme?

J.-C.G. Oui et non, en fait, j’ai été une sorte de compilateur qui a pillé allègrement les recherches qui ont été faites par les autres. Je suis parti de ce qu'il y avait comme matériaux disponibles. II y a plusieurs 
années ca se résumait uniquement à Jean Bêraud, maintenant il y a de plus en plus de recherches et par recoupement j'ai tenté d'élaborer une synthèse personnelle. La chose importante, la chose différente. c'est que lhistoire du théátre intéresse le public et ceci dans la mesure où elle devient signifiante pour nous, les gens de thestre. Pour moi, I'histoire du théatre au Québec était extrêmement importante parce que je suis incapable de dire que tout commence avec nous, que ale théatre commence avec Gélinas ou avec Tremblayn. Non, le thétre québécois est né avec Tremblay mais il était déjà vivant depuis très longtemps, c'est-à-dire le début du XIXème siècle. En fait, je considère que nous sommes tout à la fois l'aboutissement d'une tradition thétrale en partie populaire et en partie littéraire, et le début d'une dramaturgie nationale autonome et nord-américaine. C'est la raison pour laquelle je me suis intéressé à la recherche historique en rapport au théâtre, et a la recherche historique comme telle aussi. parce que le theâtre que je veux faire. c'est celui du passage à l'Histoire. Le thếtre québécois a trés souvent été a-historique, en ce sens qu'il était basé uniquement sur l'étude de la cellule familiale en dehors de ses rapports avec r'Histoire. Je crois que la problématique culturelle et politique du Québec depuis le début du XXème siècle, c'est que le Québec marque le pas, sur place, devant les portes de l'Histoire moderne, et au niveau du théâtre, à ce moment-là, l'entrée ou l'accession de l'individu, la famille ou la collectivité à l'Histoire devient le moteur dramatique le plus puissant. En tout cas, pour moi, il est plus inspirant que le moteur psychologique traditionnel.

B.A. Quand je parlais d'anti-intellectualisme, je pensais plutôt à ta méfiance a l'égard des discours portés sur la chose théatrale. Dans Jeu, tu affirmes qu'il te semble illusoire de vouloir faire appel d'autres langages pour expliquer celui des images, spécifique au théatre. «e fais plus confiance aux images qu'aux concepts * dis-tu ${ }^{2}$.

J.-C.G. Le danger que je percois dans le discours critique que les universitaires ont élaboré, ces dix dernières années. à l'entour de la chose théatrale. c'est que l'approche sémiotique ou structuraliste découpe la réalité vivante du théâtre en un champ critique forcément abstrait et qu'éventuellement ce champ-là existe par lui-même et finit par être completement coupé de la réalité vivante et complexe qu'il voulait étudier au point de départ. Le théstre ne se sent pas plus à l'aise dans une codification par trop scientifique que la réalité quotidienne dans un ordinateur programmé par un technocrate.

B.A. Tu ne penses pas que les analyses du thétre qui se faisaient traditionnellement ne rendaient pas compte de la représentation alors qu'à l'heure actuelle. l'avantage justement de la sémiologie de la représentation du théâtre c'est qu'elle tient compte non seulement du texte mais aussi de la façon dont il est mis en scène et de ces signes que sont l'atmosphère, les couleurs, les bruits, le mouvement; on essaie évidemment d'une façon un peu arbitraire de l'encoder avec 
des termes barbares mais on est davantage sensible, avec cette analyse sémiotique, à cette représentation qui était auparavant complètement laissée de côté.

J.-C.G. Je crois que l'aventure critique comme telle est très intéressante, le fait qu'un regard soit plus complexe, plus sensible et plus subtil n'est qu'une preuve de l'intelligence critique. L'erreur pour moi dans cette critique-là, ce n'est pas tellement quand les critiques la font, mais quand les praticiens de théâtre la lisent, la croient et qu'ensuite, ils essayent bètement de faire leur théâtre en fonction de ces lectures critiques plutôt qu'en fonction de la réalité vivante dú théátre, c'est peut-être là qu'on a un problème plus grave, quand on confond le phénoméne avec l'explication du phénomene et qu'on tente de reproduire l'explication plutôt que de faire naître la chose. D'ailleurs. les gens viennent de plus en plus au théâtre imprégnés de ce langage-là et c'est curieux a quel point, il peut influencer la perception du spectateur: par exemple, je me rappelle un étudiant en avionnerie qui était venu me demander, aprés le spectacle, si je pouvais lui expliquer ce que ca voulait dire quand les lumières rouges et vertes s'allumaient (rires de J.-C.G.); on lui avait confié comme travail d'étudier l'éclairage, c'était la première fois qu'il mettait les pieds dans un théâtre, la première fois de sa vie qu'il voyait un spectacle et toute son aventure a été gảchée parce qu'il a passé la soirée à regarder les projecteurs s'allumer et s'éteindre. Dans cette perspective, le théâtre devient l'illustration du discours critique. Et c'est là, pour moi, que réside le danger: quand les auteurs ou quand le théâtre lui-mème essaye d'être conforme à sa critique.

\section{La gymnastique intellectuelle}

B.A. Tu es aussi pédagogue, tu enseignes, quel est ton point de vue : est-ce que tu enseignes le théâtre ou la littérature en général?

J.-C.G. J'ai toujours enseigné ce que j'ai appelé la "gymnastique intellectuelle*, pour la bonne raison que dans une école de thétre, je cherchais a être aussi important que les gymnastes. Dans les b́coles de thêttre, on estime traditionnellement que le corps de l'acteur est ce qu'il y a de plus important et que ce qu'on trouve parfois en haut des sourcils n'est pas aussi essentiel; donc, je voulais avoir un statut, alors je me suis appelé gymnaste, mais mon cours a toujours été étiqueté comme un cours d'histoire du théâtre. Si jeen suis venu à la notion de gymnastique intellectuelle, c'est aussi parce que je me suis rendu compte que les blèves n'avaient aucune notion d'histoire générale: or comme en histoire, on explique les événements par référence à d'autres événements, j'en aurais été, très rapidement, réduit à expliquer l'inconnu par de l'inconnu encore plus inconnu et finir par parler tout seul. Dans les circonstances, je me suis dit que cela serait sans doute plus efficace de partir du vécu et de l'expérience d'acteur de 
chacun pour appréhender les mécanismes de la pensée et la relativité des formes de pensée en parallèle avec les mouvements de l'histoire: donc finalement, $d$ aborder les divers processus de la dialectique plutôt que le déroulement événementiel de la chronologie historique. Par rapport au théatre et d'autant plus par rapport aux acteurs, ca me semblait important parce que tout le monde ici au Québec est encore assez monothéiste, je dirais manichéen, avec une pensée binaire qui oscille entre le bien et le mal, le bon ou le méchant, tout comme dans une salle, le public cherche à savoir quel est le message de l'auteur, quel est le personnage qui dit ce qu'il faut penser sur la piece. alors que je considère que le théâtre est un instrument merveilleux où on peut voir quelque chose qui ne vous donne pas de réponse mais qui pose un problème auquel chacun pourrait trouver une réponse - la sienne.

Dans cette perspective, il était très important pour moi que les comédiens abordent la relativité des idées ou des croyances. Puis, la gymnastique intellectuelle, au niveau de l'enseignement s'est élargie, et j'ai crés une section d'écriture dramatique pour travailler avec les auteurs au niveau artisanal, avec une démarche pédagogique très particulière et assez unique, parce que, cette fois-ci, je considérais que la première condition pour un auteur de théatre c'était d'abord d'être un artisan du théâtre, en somme de faire partie de la maison. Il fallait se libérer de l'image de l'écrivain qui écrit chez lui assis dans un fauteuil comme Musset. Au thétre. il n'y a quiun seul moyen d'apprendre les choses, et ce n'est pas en les observant, mais en les faisant, et l'auteur de théatre a l'intérieur de l'Ecole Nationale, celui du cours que je donne, ne suit pas les cours en observateur mais participe à ceux de la section d'interprétation aussi bien que de la section technique: en trois ans, il a mis la main à la páte à tous les niveaux du théatre. Le meilleur moyen pour un auteur d'apprendre qu'un texte est trop long ou de le comprendre quand un acteur le lui dit, c'est d'avoir déjà eu soi-même le plaisir de poireauter en scène. Éalement aussi par rapport à la technique, les gens lui demandent très souvent des choses impossibles à réaliser: on lit parfois des notes de scène extraordinaires où les auteurs écrivent par exemple: l'atmosphère se transforme et l'éclairage nous fait passer de l'été à l'automne - peut-être que d'avoir étudié la technique apprend aux auleurs que les projecteurs s'allument ou s'éteignent, plus ou moins rapidement, mais qu'ils ne font pas du cinémal

Actuellement, ce qui est essentiel pour un auteur de théâtre c'est de connaître le théstre, la réalité physique du lieu théatral, parce que toute sa formation est audiovisuelle, c'est une formation issue soit du cinéma, soit de la télévision. II faut apprendre la réalité de la scéne pour devenir scénique. et c'est ce que je cherche a faire: former des écrivains scéniques, des gens qui utilisent les éléments dont ils disposent, même les contraintes, pour écrire une cuvre. Aussi, ca 
me semblait une solution économique puisqu'au Québec, un auteur de théâtre ne peut être monté et du fait mème ne peut rejoindre un public, que dans la mesure où il est capable de s'adapter aux conditions économiques actuelles de la scène québécoise. Personne, actuellement. ne peut monter une pièce de quarante personnages, fut-elle géniale: alors pourquoi l'écrire? Ensuite, il $y$ a des lieux de théâtre qui sont spécifiques, qui offrent certaines possibilités mais pas d'autres, donc un auteur est quelqu'un, pour moi, qui s'inscrit dans son temps, pas seulement son temps politique, économique, mais également dans son temps théâtral et c'était la dimension que je voulais donner à l'écriture dramatique.

B.A. Cela recoupait-il les préoccupations que tu avais au Centre d'essai des auteurs dramatiques: donner des conseils à de jeunes auteurs en fonction de réalisations possibles?

J.-C.G. La section d'écriture de l'École nationale est née, en partie, de ce que j'ai appris au Centre d'Essai où j'ai découvert qu'une fois son texte soumis, ce que, régle générale, l'auteur attendait de nous c'était qu'on fasse une table ronde où l'on aurait déterminé si c'était un chef-d'cuvre ou pas. L'auteur s'attendait, alors, a ce qu'on lui dise oui ou non et si c'était oui, il s'attendait, in petto, à ce qu'on puisse lui donner la date de la première de sa pièce qui aurait dû avoir lieu de préférence au T.N.M. où la salle était plus grande, plus culturelle. Le fait d'avoir eu cette expérience-là, d'avoir assisté, d'avoir organisé. d'avoir participé à une multiplicité de tables rondes, de lectures publiques, $m$ 'a amené à en conclure que le seul moyen de pouvoir faire un travail théâtral par rapport aux auteurs, c'était de le faire dans un cadre pratique comme celui qu'on a a l'École Nationale; parce que les auteurs du Centre d'Essai qui demandent des conseils sont assez rares, encore aujourd'hui, ils s'attendent plutôt à ce qu'on les monte ou pas: ce qui $m$ 'apparaît aujourd'hui un peu irréaliste: de production en production, le théâtre québécois a établi un certain taux de qualité et on ne peut plus aborder des thèmes qui ont déjà été traités de facon péremptoire et définitive, on ne peut plus les reprendre et cela oblige les nouveaux auteurs à connaître de mieux en mieux le métier et a faire des pieces qui ne sont plus toujours des ébauches ou des premières pièces: le public accepte de moins en moins les maladresses d'écriture, il s'attend à juste titre, à une maitrise professionnelle.

B.A. Est-ce que ce travail de préparation de nouveaux auteurs est plus facile à faire à l'École Nationale qu'au Conservatoire dans la mesure où l'École présenterait une plus grande ouverture sur les choses d'ici?

J.-C.G. Le Conservatoire, conformément à son modele parisien, a combattu d'arrache-pied le thétre québécois et en fait maintenant à l'occasion et de facon exotique. Un exercice public ne fait pas le printemps.

Depuis 1971. l'École Nationale était axée sur la création québécoise, c'était la politique de base avec le résultat qu'en neuf ans, elle a créé 
vingt-neuf pièces originales, mais c'est aussi une école nationale au sens fédéral du terme, donc mi-anglaise, mi-française, ce qui fait que la spécificité québécoise y a une signification moins grande que dans un Conservatoire qui relève d'un ministère qui s'ouvre à peine, et encore avec réticence, à la dramaturgie québécoise.

Dans le contexte du Conservatoire, le fait de s'ouvrir au théâtre de création québécois aurait impliqué un changement radical de politique el comme au niveau culturel, on reste encore extrêmement traditionnel, altaché à des valeurs étrangères, provincial dans l'âme. la possibilité de le réaliser dans le cadre du Conservatoire était impensable et le demeure sans doute encore... Quand je reçois des curriculum vitae des finissants, je remarque que les acteurs ont fait 27 pieces dont une seule québécoise... Je suis déjà allé donner une conférence au Conservatoire, à I'invitation de Gilles Marsolais, une conférence d'information, pour leur dire qu'ii existait d'autres pieces que $L e$ cemps d'une vie dans le répertoire québécois; ce n'est donc pas dans la dynamique du Conservatoire dont le principal débouché reste Radio-Canada et la post-synchro.

Ecrire. jouer, publier

B.A. Maintenant si l'on passait à la relation de J.-C.G. avec les livres, avec l'écrit, compte tenu que cette entrevue est faite dans le cadre d'une revue universitaire... Comment expliquer le fait que nombre de pièces de J.-C.G. sont publiées, et qu'il $y$ apporte un soin extrême?

J.-C.G. En lait, il n'y en a pas beaucoup de publiées parce que i'y attache beaucoup de soin, c'est une élégance, presqu'un côté précieux chez moi, j'aime beaucoup la littérature alors quand je publie une pièce surtout compte tenu des conditions de publication, c'est-à-dire qu'on ne publie pas de versions remaniées, je suis donc condamné à un texte définitif.

Publier une piece avant qu'elle soit jouée, pour moi, ce n'est pas tenir compte de la réalité théatrale, car si on fait des coupures ou des changements dans une piece, c'est parce qu'il fallait trouver une meilleure solution, donc, a mon avis, il ne faut la publier qu'une fois qu'elle a connu l'expérience de la. scène, de plus avec le recul cela nous permet au moment de l'écriture définitive de régler certaines choses qui parfois ne l'ont pas été dans le cadre du spectacle, où parfois on trouve une solution théâtrale mais pas nécessairement une solution textuelle. Lorsquion s'asseoit pour publier un texte de théatre, c'est une partition que l'on publie, parce qu'il faut qu'une tierce personne puisse la lire. la comprendre et la traduire scéniquement en fonction de ce qu'elle veut véhiculer. Donc, pour moi, à ce moment-là, c'est un problème de notation, de musicien qui m'est posé. C'est pourquoi mon rythme de publication est trés en retard sur mon rythme de production: j'accorde beaucoup d'importance a la publication mais c'est une vanité de littérateur. 
B.A. Est-ce que l'esprit dans lequel c'est fait permettrait que ces pièces soient jouées par d'autres que J.-C.G., ce qui ne se produit pas souvent?

J.-C.G. Dans les dernières années. il y en a eu quand même certaines qui ont été rejouées. Ce n'est pas rejoué à Montréal, puisque mon activité principale se déroule à Montréal, nécessairement c'est à nous de les rejouer, elles font partie du répertoire du Théatre d'Aujourd'hui. Par contre, elles ont été jouées à Québec, à Sherbrooke, à Ottawa, à Toronto. dans la Gatineau.

B.A. Des troupes indépendantes du Théâtre d'Aujourd'hui?

J.-C.G. Oui, et à ce moment-là, c'est extrêmement intéressant pour moi, parce que je vais voir une autre version et je dirais. règle générale, qu'elle est conforme à l'esprit dans lequel elle a été taite, avec une vision différente.

B.A. Pour revenir aux publications, quelle est l'utilité du livre pour le dramaturge?

J.-C.G. Pour un écrivain de théâtre, la fonction du livre n'est pas la même que pour un romancier, c'est une fonction de partition, le livre est valable dans la mesure où la partition est la plus exacte, la plus explicite possible et permet a des gens de la reproduire scéniquement en accord avec les idées originales, tout en permettant à chacun d'y ajouter une créativité nouvelle, d'avoir un regard critique qui est nécessairement différent du fait qu'on présente la pièce 5 ou 6 ans après sa création. ce qui fait qu'inévitablement, on met l'accent sur certaines choses plutôt que sur d'autres.

B.A. La publication d'une pièce en fait une pièce de répertoire, de répertoire québécois, par exemple?

J.-C.G. Oui, mais te répertoire ce n'est pas seulement la publication qui le détermine, c'est le degré d'intérêt pour la piece et le fait qu'on la rejoue ou pas. Ou elle fait partie d'un répertoire vivant, ou on la range sur une tablette dans une bibliotheque. Ma relation avec le livre est une relation avec l'objet-livre et mon plaisir d'écrire pour faire un livre n'est pas le même que celui que j'ai dans mes rapports avec le théătre. Si je prends le temps de faire des livres, c'est parce que cela m'amuse, et que i'y prends un plaisir autre, tout a fait différent pour moi de celui du théâtre, puisque c'est important, pour mon plaisir. que le livre soit beau, bien présenté...

Y.L. L'objet...

J.-C.G. Absolument. L'objet-livre qui plaît à l'amateur de livres, à celui qui, en moi. aime les livres avec passion. Bien sûr que j'aime voir mes pièces publiées pour le texte, mais aussi pour que je puisse tenir un bel objet dans mes mains, que je puisse le feuilleter avec sensualité. Je bouquine depuis que j'ai l'ảge de six ans, forcément, face aux livres. j'ai la main et l'ceil critiques. 
Y.L. C'est dans ce registre que tu travailles avec Victor-Lévy Beaulieu, avec l'éditeur VLB?

J.-C.G. Avec Victor-Lévy Beaulieu, j'ai fait partie de la maison d'édition et il y a aussi une altinité au niveau de la pensée, des différentes aspirations littéraires, au niveau des auteurs qui s'y rencontrent, un genre de carrefour. Et le fait aussi que Lévy fait de beaux livres, alors il y a une partie de moi, celle qui aime les livres, qui est comblée.

Y.L. Par rapport cela, comment peux-tu te sentir : s'il existe un livre, tout de suite l'université s'en préoccupe, cela devient quelque chose sur quoi on peut travailler?

J.-C.G. C'est une autre piste. Pour moi, il y a une différence complète entre celui qui travaille pour le théátre et le littérateur, ce sont deux personnes différentes. II $\vee$ a d'abord en moi ce que vous appelez l'écrivain scénique, I'homme de thétre qui a tous les plaisirs. parce que ce qui est important pour moi, c'est d'abord de monter une pièce...

Y.L. Excuse-moi, tu dis qu'on t'appelle l'écrivain «scénique», mais c'est toi qui dit que tu es devenu un écrivain scénique...

J.-C.G. Oui, peut-être, c'était dans l'air ambiant, d'habitude, je me considère plutôt comme un homme de théâtre, parce que je ne vois pas en quoi ma fonction est differente de celle d'un homme de theátre classique. je veux dire historiquement. Le fait que j'écrive en plus, c'est une corde de plus, c'est peut-être la seule dillérence qui existe entre l'homme de théŝtre classique et moi. Aegle générale, c'est une tradition théátrale d'être un actor-manager. I'homme de théâtre joue, fait de la mise en scène et dirige le théâtre. Moi je fais de la mise en scène, j"écris et je dirige un thétre. Dans les temps modernes, on peut penser à Brecht. Ecrivain "scénique» après tout est peut-être une expression qui correspond assez bien.

Y.L. II me semble que tu utilises l'expression pour l'opposer à l'écrivain littéraire?

J.-C.G. D'une façon oui. J'aborde le théâtre en fonction du théâtre, ce que j'en retire c'est un plaisir théatral, avec toutes les contraintes, les limites et les joies qui siy trouvent. Pour faire un livre, je fais appel a une autre partie de moi-même, qui est au deuxième plan quand j'écris pour le théâtre, c'est-à-dire que ce n'est pas la littérature qui m'intéresse sur scène, c'est l'efficacité de ce que j'ai écrit, c'est-à-dire que la présentation visuelle corresponde à ce que j'espérais, que le jeu me plaise ou corresponde à ce que je recherchais, ceci dit, il est bien évident que la pièce doit être bien écrite c'est un sine qua non. Mais ce n'est pas le côté littéraire qui me fascine, tandis que lorsque j'arrive au niveau de la mise en forme definitive en vue de la publication, je me permets d'y accorder la priorité. 


\section{La création collective}

B.A. Toujours dans le domaine de l'écriture, pour aborder le problème de la création collective versus l'écrivain seul, on a commencé avec Les Enfants de Chénier par de la création collectıve, comment cela se passait-il, qui écrivait finalement?

J.-C.G. J'ai toujours rempli la fonction d'écrivain. j'ai toujours fait la mise en scène. Une confusion assez énorme s'établit maintenant au sujet de la création collective. A ce moment-là, du moins pour moi, c'était une rédéfinition des différents rapports qui existaient entre les artisans de théâtre, ce n'était pas nécessairement une confusion des rôles. Ca m'amuse un peu, parce qu'aujourd'hui. je travaille parfois avec des gens, des collectifs, qui eux font des différences sémantiques, de vocabulaire... ils ont un metteur en scène qu'ils appellent $\alpha$ observateur , les fonctions sont toujours là mais on leur a donné des noms différents. Notre attitude au moment des Enfants de Chénier quelle était-elle? Le problème qu'on avait c'était d'essaver de trouver une forme théâtrale. des formes nouvelles car d'un côté il $\vee$ avait des comédiens qui cherchaient un renouveau et de l'autre, moi qui voulais tenter une approche théâtrale différente.

Ce qu'on recherchait, c'était de créer un nouveau type de réalisme en scène, d'avoir une réalité de jeu qui correspondait à l'acteur québécois et qui, au niveau du dire scénique, correspondait a la façon dont on est ici. Donc, on a utilisé l'improvisation et principalement l'improvisation verbale qui permettait à la fois de débloquer le naturel au niveau de la langue parlée comme telle mais aussi parce que la langue, ce n'est pas seulement des mots mais toutes les émotions qui sont liés aux mots, donc, c'était également de retrouver une gestuelle, un langage du corps, une façon d'ètre en scène qui corresponde à ce qu'on est au Québec. Pour moi, c'était également de travailler avec des images culturelles qui étaient les nôtres et qui créaient un consensus immédiat dans le public. La conjoncture nous a fait travailler de cette manière. En somme. nous cherchions une langue théâtrale populaire. Dans la création collective, je remplissais la fonction de l'écrivain scénique, je concevais la mise en scène et j'assumais l'organisation générale du spectacle. La création collective était vivante parce qu'elle changeait constamment; dans le cadre du premier spectacle. on s'est demandé : on fait un spectacle, mais sur quoi? . La seule chose que les gens avaient en commun à l'intérieur du groupe des Enfants de Chénier, c'était qu'ils venaient tous de l'École Nationale, pas de la même classe d'ailleurs, ni de la mème époque. Donc, quand on s'est demandé sur quel thème travailler, j'ai eu l'idée de reprendre des scènes où chacun des acteurs avait connu un flop magistral, il m'a semblé que ca serait intéressant d'essayer de réussir en québécois ce qui avait été raté en français. Comme nous avions des préoccupations par rapport au théâtre classique, au thêatre 
français, au colonialisme culturel, par rapport à toute la signification culturelle que cela avait à ce moment-là, c'est devenu le theme du spectacle qui était un refus, une liquidation, un genre d'adieu définitif à ce théâtre-là et aussi pour les gens qui ont fait cette expérience. une sorte de point linal a leur formation. Un des aspects nouveaux du spectacle était évidemment les improvisations que les acteurs faisaient en scène dans un style compétitif que la L.N.I. a repris depuis et poussé a son maximum. Pour le deuxieme spectacle Diguidi Diguidi $\mathrm{Ha}$ ! $\mathrm{Ha}$ ! Ha! l'improvisation verbale des comédiens a été utilisée d'une façon exploratoire et finalement le spectacle qui s'est imposé était un spectacle sur la famille, une liquidation de la famille traditionnelle: comme point de départ $j$ 'ai utilisé l'improvisalion verbale des comédiens en employant une méthode de notation sélective quej'avais développée comme reporter-journaliste et qui consiste à ne noter que les éléments intéressants: puis ayant travaillé avec les thèmes, j'ai réorganisé une partie de la matière, créé une structure et j'ai fait une piece.

\section{B.A. Est-ce que tu jouais avec eux?}

J.-C.G. Non. J'ai toujours été le témoin, le spectateur privilégié; au niveau du travail, ma fonction a toujours été celle de l'écrivain scénique.

Pour moi, que tu partes de tes souvenirs denfance, d'une recherche dans les journaux, que tu partes de l'improvisation des comédiens, d'enregistrements faits au magnétophone, de documents, de statistiques Canada, cela n'a aucune importance puisqu'il faut toujours que lu transformes cela en écriture scénique, c'esı le travail de l'écrivain de toute manière de prendre un matériau brut et de le transformer en écriture théâtrale; la fonction de l'auteur de théatre était donc redéfinie parce qu'a ce moment-là, on ne partait plus du texte, mais on y arrivait, c'est une différence dans l'approche qui est fondamentale car à cause d'un complexe culturel et du complexe du texte écrit. les acteurs avaient par tradition l'impression de recevoir le texte, le texte sacré même puisque c'était, règle générale, un texte classique; en somme. on recevait un texte quasiment venu de Dieu et on oubliait qu'au niveau du théâtre, le texte, c'est la dernière chose qui doit naitre. C'est-à-dire qu'au début, pour l'auteur, il y a une situation, la parole nait des émotions, donc il tallait, pour l'acteur, retrouver cette mécanique arriver au texte: le texte est un aboutissement, pas un début.

Règle générale, pour façonner un spectacle. on partail de thèmes; mais jai établi comme théorie puis comme pratique, que c'était mieux de ne pas faire improviser les acteurs sur le thème lui-même du spectacle, mais plutôt sur des thématiques connexes, je partais donc avec une idée auteur de laquelle les acteurs improvisaient mais je ne cherchais pas à écrire le spectacle immédiatement, plutôt à miimprégner du sujet.

Donc, lorsque jarrivais avec le texte écrit et que les acteurs avaient déjà improvisé alentour des thèmes, ca nous permettait de monter 
le spectacle en deux semaines puisque tout le monde savait dans quel esprit travailler mème si le texte n'était pas une transcription de ce qui avait été improvisé.

Pour le troisième spectacle Si Aurore m'était contée deux fois, on a encore fait des improvisations un peu au niveau des themes mais surtout au niveau des mécanismes de jeux. Sauvageau a improvisé des sermons par écrit mais je suis arrivé avec un spectacle organisé. Sur scène, on n'utilisait plus l'improvisation verbale mais musicale, chantée. Après. il y a eu d'autres spectacles où j'ai écrit de façon stricte sans faire d'improvisation, tranquillement, pour moi; l'improvisation devenait une technique de comédien, de metteur en scène. non pas une technique décriture. De plus en plus, les fonctions a l'intérieur du groupe que l'on formait et le style scénique se définissaient: par exemple au niveau visuel, il fallait tenir compte de nos moyens limités; quand on est pauvre, il y au moins une chose qui est riche, c'est la couleur, rapidement un style scénique qui nous était propre est apparu et celui que je pratique aujourd'hui est en grande partie issu de celui-là, parce qu'on a utilisé deux choses: la couleur vive et la lumière, la lumière était crue, music-hall, parce qu'on n'avait pas les moyens de patiner des décors. de toute façon, je n'aimais pas ca; mais en plus cela nous permettait d'avoir un visuel vibrant et contemporain. En somme, la lumière a pris de l'importance a l'intérieur du style. de la facon d'aborder le réel scénique parce que la couleur était notre seule richesse, la richesse des pauvres.

Puis le lieu où on jouait, au Théâtre d'Aujourd'hui, dont les contraintes physiques nous permettaient de travailler dans la proximité avec le spectateur, ce qui a influencé énormément le jeu et l'écriture, mis l'accent éventuellement sur les costumes plutôt que sur le décor et fait de la lumière, l'agent principal de ce qui est devenu aujourd'hui un style de théatre qui nous caractérise.

B.A. Pour revenir à la création collective, c'est donc à partir de la troisième piece que J.-C. Germain aurait repris les rènes pour écrire tout seul des pieces? Comment passe-t-on d'un type de création a l'autre?

J.-C.G. Pour moi, la création collective fut une tentative de redéfinir ou de définir les rapports qui doivent exister à l'intérieur de la cérémonie théâtrale. Prenons un exemple. celui de la musique : au début, on partait des souvenirs, de l'héritage musical de chacun, tout le monde peut pousser une toune, et on a fait des tounes de folklore où chacun chante seul, au plus collectif, en canon, ou à répondre. Le moment est arrivé où pour organiser-une matière musicale de façon plus complexe. it a fallu faire appel à un musicien. la matière musicale étant devenue trop complexe, on avait besoin d'un compositeur.

La même chose s'est produite au niveau du décor et des costumes, au début c'était purement une ambiance, une atmosphère, une scénographie rudimentaire, mais ensuite la nécessité d'une conceptualisa- 
tion visuelle globale s'est imposée et en même lemps celle d'un décorateur et d'un costumier. Plus tard, une maquilleuse, un choré- graphe, un concepteur sonore.

Pour moi, la création collective a été d'une certaine façon un moyen de réinventer le théâtre et de rédéfinir les fonctions de chacun, car tout cela était sclérosé. Ca $\mathrm{m}$ 'a aussi permis de définir clairement les fonctions d'euteur et de metteur en scene qui sont pour moi deux choses très différentes. Quand jeécris, je tiens compte de l'espace de jeu, je suis un écrivain scénique, je tiens compte ausși de toutes sortes de données pratiques, comme les changements de costumes, les acues* d'éclairage, parce que lorsque j'arrive en répétition. je deviens un metteur en scène. C'est au metteur en scène que les problemes sont posés et c'est à lui de les résoudre, s'il n'arrive pas à trouver une solution scénique, il se retourne vers l'écrivain et lui demande de refaire autrement ce qui cloche, donc c'est le metteur en scène qui prime au niveau du spectacle. L'écrivain ne peut exiger le maintien d'un passage textuel parce que c'est d'une importance littéraire quelconque. le metteur en scène s'y refuse, parce que ce qui est important c'est que le spectacle passe dans son ensemble, qu'il réponde a ce quion attend de lui, avec le résultat assez cocasse que par exemple, dans le contexte de La Diva, ce que le metteur en scène a dû couper à l'écrivain n'étaient pas les choses les plus mal écrites. au contraire même, c'était trop bien écrit et en termes de représentation théâtrale, trop bien écrit pour le personnage, assez littéraire pour que le public entende la voix de l'auteur plutôt que celle de La Diva. Je fais la diflérence entre ces deux fonctions parce quelles impliquent deux points de vue. Mais quand le rideau s'ouvre, une seule chose doit exister: te spectacle.

B.A. Alors, pour revenir a l'émergence de l'écrit scénique à partir de la création collective, ce doit être une sorte de naissance difficile, le moment où il faut décider qui va écrire. Car il ne s'agit pas d'imposer un texte mais de partir quand même dimprovisations de comédien/ne/s...

J.-C.G. Personne au théâtre ne peut imposer un texte; ma pratique du thétre m'a appris que le premier public d'un auteur. ce sont les comédiens avec qui il travaille, si ces 7 ou 8 personnes. si le décorateur, l'éclaira. giste. le costumier. chacun dans sa fonction, ne sont pas intéressés à la pièce d'un auteur au départ, il n'y aura pas grande chance d'y intéresser un public. à aucun niveau, l'auteur de théâtre ne peut prétendre à la primauté du texte de droit divin. Un texte est joué tel qu'il a été écrit parce que c'est la meilieure solution textuelle possible dans les circonstances particulières d'un spectacle donné: pour moi, il ne peut $\checkmark$ avoir d'autres raisons.

B.A. Mais on ne peut pas frustrer un comédien qui avait eu une idée ou une intuition au moment de la création collective et qui ensuite ne la retrouve pas ou la retrouve différemment chez la personne qui a pris en charge lo pièce. 
J.-C.G. Le théâtre est un art collectif, par nature, une suite de compromis, tout le monde doit mettre de l'eau dans son vin; personne, dans une production théâtrale, ne peut exercer sa fonction sans tenir compte de tout le monde, pas plus l'auteur d'ailleurs que le décorateur ou l'éclairagiste ou le musicien, tout comme le metteur en scène qui ne peut orchestrer sa partition qu'en fonction des capacités de jeu et de compréhension des comédiens.

Le théâtre est un art du possible où tout est fait pour arriver à un produit éphémère qui est issu de la concertation relative des différents créateurs, personne ne peut prétendre à une vision qui va s'exprimer en solo, d'autant plus qu'une fois en représentation, seuls les acteurs ont un contact direct, réel, avec le public.

B.A. Cela se comprend par rapport à l'écrivain scénique mais par rappart au comédien, dans la phase création collectivex, s'il y en avait un qui disait non seulement «ç, c'est ma vision a moi * mais «c'est mon bout à moi $\gg$ ? \& c'est ma scène $\nsim$ ?

J.-C.G. J'ai remarqué, dans le cas de ceux qui travaillent en collectif, qu'on a parfois tendance à donner plus d'importance a la manière dont un texte a été écrit qu'au texte lui-mème; si les gens se sont mis à 15 pour écrire un texte et ont senti le besoin de partir en bateau à voile puis de passer par les Bermudes pour le faire, ca releve de l'anecdote, mais ce qu'on va juger comme spectateur, ce n'est pas tellement la manière dont on s'y est pris pour écrire un texte ou le fait qu'il témoigne du vécu personnel des acteurs. c'est la cohérence du spectacle. Très souvent. d'ailleurs, j'ai constaté que le résultat dramatique est tributaire de la mécanique de fonctionnement d'un collectif: une bonne partie des créations collectives sont faites à partir d'un schéma qui reproduit la structure mème de fonctionnement du groupe, une suite de solos, ou de monologues, ou de scènes à deux tronquées, c'est-à-dire que c'est un monologue mais il y a un contre-pittre qui donne l'impression que c'est un dialogue, le tout réuni, cousu ensemble, par des chansons. $\mathrm{N}$ 'importe quelle pratique de théatre assez longue vous amène a aborder des problèmes techniques et dramatiques de plus en plus complexes, et à ce moment-là, tant au niveau de l'écriture qu'au niveau de la réalisation. c'est comme pour la musique: n'importe qui peut pousser une toune mais pour trousser une symphonie ou un concerto. il faut d'abord, se donner les moyens de pouvoir l'écrire.

B.A. Dans certains cas, si les comédiens ont l'intuition, mais que tu n'es pas capable de reprendre ca, de l'organiser...

J.-C.G. N'importe qui peut improviser n'importe quoi et c'est dans la mesure où quelqu'un est ignorant des difficultés inhérentes à une technique donnée que tout peut lui paraître facile; à titre d'exemple, les auteurs de téléromans, règle générale, ne savent pas écrire et c'est ce qui leur permet de pondre émission après émission. Thomas Mann a déjà dit que c'était difficile d'écrire uniquement pour les écrivains. Je respecte 
le métier de comédien et encore plus ceux qui le maîtrisent, la moindre des choses à laquelle on puisse s'attendre, c'est que les comédiens respectent le métier d'auteur de thétre. Pas la fonction abstraite mais le degré de maitrise de celui qui le pratique.

Ce que la création collective a permis et va permettre, c'est un respect réel et mutuel des fonctions à l'intérieur du théâtre et non plus des relations basées uniquement sur l'autorité hiérarchique. Pour moi, l'auteur a la primauté parce qu'il est le maitre des situations dramatiques et des mols tout comme le musicien-compositeur est le maitre des sons. mais il ne peut pas imposer à l'acteur d'interpréter ce qui est écrit comme c'est écrit si ce n'est pas évident: les comédiens posent des questions au texte et aux personnages et si le texte ou les personnages ne peuvent y répondre, l'auteur n'a qu'à retourner à son établi et à réécrire, s'il n'est pas capable de faire ca, qu'il se fasse publier sans être joué.

\section{Thestre ot sociotes}

Y.L. Est-ce que le recours à des comédiens ancrés dans leur propre culture ne permet pas aussi de toucher plus facilement un archétype québécois?

J. -C.G. Au moment où j'ai abordé cette façon de travailler avec les comédiens. ça correspondait à une période où la société québécoise comme telle cherchait à redéfinir toutes ses structures politiques. elle avait des comités de citoyens qui voulaient se faire entendre et participer aux décisions collectives, c'est-à-dire qu'à ce moment-là, il y avait à l'intérieur de la société une redéfinition et une redistribution du pouvoir, Il v avait aussi la naissance du PQ qui cherchait à créer un type de parti politique différent où la participation démocratique serait opérante. Ce qui se passait au niveau du théatre, au niveau de la redélinition et de la redistribution du pouvoir créateur, était en concordance parlaite avec l'ávolution de la société québécoise. Le thétre n'existe pas uniquament par ses inpports avec le texle de la représentation. nou. In représantation, comme la texte. sonl a l'imago de tout un fonctionnement organique el quand les gens viennent ici au Thétre d'Aujourd'hui, ce qu'ils reçoivent sur scène est oussi une image de tout le fonctionnement du Théatre d'Aujourd hui et c'esı ce qui fait que c'eșt très différent de ce qu'ils perçoivent au Fidesu Vert, au T.N.M. ou au Conventum, il $v$ a lout un arrière-plen dillérent et je crois que la pratique théatrale, la façon dont le pouvoir créateur est réparli à l'intérieur d'une maison de théatre, a une grande influence sur le produit, même plus, que le produit en est l'expression même et l'auteur n'en est alors, qu'une des parties constituantes.

Y.L. Est-ce que ça peut aller jusqu'à l'expression spontanée d'un imaginaire collectif qui se manifeste plus facilement du fait que plusieurs personnes improviseraient librement sur un theme? 
J.-C.G. Une société peut sentir le besoin d'écrire d'une certaine manière parce qu'elle a le besoin d'une expression plus collective: à d'autres moments, une autre société peut se reconnaître a travers une voix qui perle au nom des autres. De toute façon, la fonction de l'écrivain de théâtre n'a de valeur et d'intérêt que dans la mesure où il est une voix qui parle pour les autres, s'il ne parle qu'en son nom propre il n'intéresse personne, a moins qu'il ne soit un cas pathétique mais on voit une pièce pathétique pas vingt-cinq du même auteur. Consciemment ou inconsciemment, la représentation théâtrale est toujours a l'image du systeme collectif; je me rappelle avoir vu un spectacle du Théatre noir de Prague, c'était fantastique, l'incarnation mème de leur système, c'était insignifiant, absolument, avec des moyens techniques considérables parce que la Tchécoslovaquie est un pays moderne mais aussi un pays de l'Est où le comédien met un travail fou au service d'une histoire qui ne signifie absolument rien; pour moi, toute la problématique tchéque était sur la scène. un contenant tape-à-l'ceil sans contenu, une société moderne réduite à la pantomime comme le furent les acteurs au Moven Age, un grand silence agité.

Pour un touriste qui vient nous voir, qui entre dans une salle de théâtre. il reçoit de façon globale une connaissance de la société québécoise et de son fonctionnement, de même façon qu'en France, on sent toute la société française à travers sa représentation théâtrale; il n'y a pas de quoi s'étonner de la prédominance absolue du metteur en scène quand on pense à l'héritage, non liquidé. de de Gaulle.

Y.L. Et maintenant tu ne travailles plus comme ca? Est-ce que cela veut dire qu'il y a des transformations?

B.A. Des transformations corrélatives de la société québécoise? On pourrait peut-être conclure là-dessus: qu'est-ce qui fait la spécificité du Théatre d'Aujourd'hui, aujourd'hui? Voila dix ans, la création collective pouvait encore refléter certaines aspirations culturelles et politiques: redistribution des rôles et des initiatives. Montée d'un parti provincial nationaliste enclin a la cogestion, préjugé favorable aux travailleurs. etc... mais depuis 1976 et surtout 1980, la situation est tout autre. Peut-on mettre en perspective un certain raidissement du pouvoir avec le retour au texte d'auteur et le déclin de la création collective?

J.-C.G. II y a des choses qui se passaient au Québec et qui ne se passent plus. Tout l'arrière-plan politique, toutes les tentatives de redéfinir le pouvoir n'ont plus la pertinence qu'elles avaient; soit qu'elles se sont tues apres 1970. soit qu'elles se sont traduites, après l'arrivée du PQ au gouvernement, en une série de mesures technocratiques: a la limite, ceux qui ont souhaité les changements sont maintenant les premiers à en combattre les retombées bureaucratiques; la démocratisation formelle du pouvoir a produit une machine étatique encore plus lourde, omniprésente et autocratique. Le Théatre d'Aujourd'hui se definit toujours en fonction de son point de vue qui est critique et 
de sa raison d'être qui est l'établissement et le maintien d'une dramaturgie québécoise; aujourd'hui après avoir créé une permanence administrative et installé une structure de production efficace, nous nous retrouvons aux prises avec un problème qui semble être celui de tout le Québec: I'Etat technocratique. Comment demeurer vivant, autonome, et créateur tout en devenant progressivement, bien malgré nous. l'être abstrait et anonyme qe l'Etat souhaite de plus en plus comme interlocuteur.

Y.L. Et ca peut eller jusqu'au fait que tu écrives maintenant tes pieces tout seul?

J.-C.G. Face à la page blanche, l'auteur de théâtre, comme l'écrivain, est toujours solitaire. Ceci dit, la relation que j'ai créée avec les comédiens, avec mes collaborateurs, avec tous les gens avec qui je travaille depuis des années est toujours la même. c'est une question de modalité. j'écris toujours une pièce pour des comédiens en particulier. pour des gens que j'aime bien, dont je connais les possibilités et avec lesquels je peux ' $m$ 'amuser, à ce moment-là, on recrée le plaisir de jouer du début, comme au début, pour moi il 'n'y a pas de changement quant à l'approche. Mon écriture est d'abord et avant tout théâtrale, en fonction d'un lieu scénique spécifique, de certaines possibilités techniques: donc, dès le point de départ, quand je connais le nombre de comédiens que le budget me permet, je les choisis en fonction du theme que je veux traiter et la pièce naît en fonction des cinq ou six comédiens que j'ai réuni, avec d'autres comédiens la pièce serait autre.

L'idée initiale d'avoir une compagnie permanente est tombée pour des raisons d'ordre économique et social, c'est-à-dire que les comédiens professionnels ne peuvent fonctionner de cette façon-là à moins de changer de statut parce que les comédiens professionnels gagnent principalement leur vie à la télévision, au cinéma, a la postsynchro, le fait d'être permanent dans une compagnie les empêche de bénéficier de ces possibilités et de boucler leurs fins de mois; chaque fois qu'un comédien accepte un contrat extérieur à la compagnie permanente. il remet en question sa participation au groupe, dans les circonstances, il peut se sentir a juste titre brimé par le groupe et le groupe frustré par sa désaffection temporaire. en fait, tous les problèmes découlent du fait qu'il est impossible de maintenir économiquement une compagnie permanente formée de comédiens professionnels.

B.A. II n'en reste pas moins qu'on garde un esprit maison, un auteur maison; on a un théâtre: un public; on a le débouché que représente aussi le Théâtre d'Aujourd'hui par rapport à l'Ecole nationale de théâtre pour certains étudiants?

J.-C.G. La vocation et la politique du Théâtre d'Aujourd'hui sont claires, c'est le seul thêtre qui se consacre exclusivement a la création d'ceuvres québécoișes; dans les circonstances, c'est plus que normal qu'on y 
ait retrouvé, qu'on $y$ retrouve et qu'on $y$ retrouvera tous ceux quı partagent la mème idée fixe.

B.A. C'est le théâtre dit ainstitutionnel *?

J.-C.G. Le Théâtre d'Aujourd'hui n'est pas un théâtre institutionnel, ni danș son idéologie, ni dans son répertoire. c'est une compagnie instıtutionnelle de théâtre: passé un certain stade. l'existence d'une compagnie est reconnue par l'Etat; de toute façon, après un certain temps, qu'on le veuille ou non, on devient une institution; de là à devenir une institution $\alpha$ institutionnelles, il y a une marge: quand le ministère des Affaires culturelles nous a affublé du vocable, il a senti le besoin d'y ajouter des guillemets, comme si l'Etat québécois avait peur den assumer le sens ou la responsabilité à mon avis, c'est un grave signe de manque de confiance, de peur du ridicule, pas des compagnies de theâtre, mais du MAC vis-à-vis de lui-même.

Personnellement, la permanence ou la continuité ne me font pas peur, c'est la raison pour laquelle j'ai travaillé à créer une machine théâtrale comme le Théâtre d'Aujourd'hui et que je suis devenu un dramaturge maison; d'abord pour bénéficier de l'instrument en tant qu'écrivain scénique et ensuite pour prouver la possibilité d'une continuité théâtrale, il y a dix ans, je voulais prouver que c'était possible de faire de la création tout le temps: ce qui fait que je ne peux pas voir le théâtre qui se fait maintenant comme une rupture, l'approche ou la pensée peuvent être différentes, mais il s'inscrit normalement dans la continuité de notre démarche créatrice: c'est la même chose avec les auteurs avec qui j"ai travaillé à l'Ecole nationale et qui font maintenant des pièces que je peux présenter: le thétre québécois se transforme, pas nécessairement dans des directions que j'avais prévu ou que je souhaiterais; certaines options $m$ 'agacent, d'autres m'ennuient carrément, mais on reste tout de même d'accord sur quelque chose de profond, une évidence qui était loin d'être aussi évidente il y a dix ans. que le theátre que l'on fait ici doit être fait pour les gens d'ici, dans la langue d'ici, sur des problèmes d'ici.

Pour diverses raisons, j'ai été amené à combattre l'idéologie théâtrale de ceux que j'ai appelé les fils du père Legault mais je dois laisser a d'autres le soin de combattre les fils du Théâtre d'Aujourd'hui: parce que j'avoue que, personnellement, je les aime bien, même quand ils ignorent complètement l'existence du Théâtre d'Aujourd'hui.

B.A. En guise de conclusion ou de boutade, on a parlé de théâtre ainstitutionne! ${ }^{\star}$, puisque le Théatre d'Aujourd'hui est classé pour les besoins de la bureaucratie dans les théâtres institutionnels, est-ce que J.-C. G. ne craint pas de devenir lui aussi une institution au Québec dans la mesure où on l'invite à des conférences, dans des colloques, où on lui consacre des dossiers, des numéros spéciaux de revues...

J.-C.G. (rires de J.-C. G.). Récalcitrant ou consentant, j'y peux rien, l'institution se fait malgré moi, tout ce que je peux y voir, c'est un défi à relever et 
dans les circonstances, tout ce que j'espère c'est de pouvoir devenir une institution qui continue de vous étonner (rires de J.-C. G., de nous-mêmes et de la statue de Louis Cyrl.

1. Voir dans le dossier de Jeu consacré à Louisetle Dussault, I'entrevue que celle-ci nous accorde $\left(\right.$ Jeu $n^{\circ} 16$ ). A noter que Jeu $n^{\circ} 13$ consacrait à l'automne 1979 un dossier volumineux à Jean-Claude Germain.

2. Voir Jeu n० 13, p. 32 (entrevue de Francine Noël et Gilbert David). 